\title{
Parâmetros de qualidade de méis inspecionados comercializados na cidade de
}

\section{Barreiras-Bahia}

Quality parameters of inspected honeys sold in the city of Barreiras-Bahia

Parámetros de calidad de las mieles inspeccionadas vendidas en la ciudad de Barreiras-Bahia

Recebido: 06/12/2020 | Revisado: 11/12/2020 | Aceito: 15/12/2020 | Publicado: 13/01/2021

Camila Filgueira de Souza

ORCID: https://orcid.org/0000-0002-1281-009X Instituto Federal de Educação, Ciência e Tecnologia da Bahia, Brasil E-mail: souza.cf94@gmail.com

Luana Regina Pereira Alves

ORCID: https://orcid.org/0000-0003-4677-846X Instituto Federal de Educação, Ciência e Tecnologia da Bahia, Brasil E-mail: luanareginaalves@gmail.com

Fabrício Luiz Tulini

ORCID: https://orcid.org/ 0000-0002-0242-2835

Universidade Federal do Oeste da Bahia, Brasil

E-mail: fabricio.tulini@ufob.edu.br

Alexandra Mara Goulart Nunes Mamede

ORCID: https://orcid.org/0000-0001-7154-6017

Instituto Federal de Educação, Ciência e Tecnologia da Bahia, Brasil

E-mail: alexandra.mamede@ifba.edu.br

Ana Celia Barreto de Araújo Santana

ORCID: https://orcid.org/0000-0002-5732-6808

Instituto Federal de Educação, Ciência e Tecnologia da Bahia, Brasil E-mail: anabarreto@ifba.edu.br

Ítalo Abreu Lima

ORCID: https://orcid.org/0000-0003-0559-3688

Instituto Federal de Educação, Ciência e Tecnologia da Bahia, Brasil E-mail: italoabreu@ifba.edu.br

\begin{abstract}
Resumo
O presente estudo foi desenvolvido com o objetivo de avaliar a qualidade de méis industrializados (inspecionados) comercializados na cidade Barreiras-Bahia, através de análises físico-químicas. Foram coletadas 5 marcas de méis inspecionados, obtidas em supermercados e mercados da cidade de Barreiras. Houve diferença estatística $(\mathrm{P}<0,05)$ entre os tratamentos para as análises quantitativas. Todas as amostras apresentaram valores de atividade de água abaixo de 0,60, embora a não seja exigida pela legislação, é um importante parâmetro para verificar o teor de água livre no produto disponível. Todas as amostras de méis ficaram com umidade abaixo de $20 \%$, atendendo a legislação. Os valores de $\mathrm{pH}$ nas amostras analisadas, variavam de 3,79 a 4,16 e para acidez ficaram entre 21,64 e 36,35 meq/kg, atendendo assim a legislação. A análise de $\mathrm{pH}$ pode auxiliar na avaliação da qualidade de méis, em conjunto com a análise de acidez. Os teores de açúcares redutores obtidos ficaram entre 70,95\% a 73,50\%, já para sacarose aparente que de acordo a legislação brasileira preconiza um limite máximo de $6 \%$ os valores obtidos variaram entre $1,85 \%$ a 4,40\%. Apenas a mostra MM5 estava dentro dos limites de (HMF), em relação a atividade diastásica, todas estavam abaixo do valores mínimos recomendados. Em relação as análises qualitativas de Lund e Lugol, todas as marcas analisadas não foram indicativas de adulteração ou má conservação do mel. As 5 marcas analisadas de méis com (SIF) atenderam a legislação nos seguintes parâmetros: umidade, cinzas, pH, acidez, açúcar redutor, sacarose aparente.
\end{abstract}

Palavras-chave: Mel; Hidroximetilfurfural; Atividade diastásica; Industrial.

\begin{abstract}
His study was developed with the objective of evaluating the quality of industrialized (inspected) honeys sold in the city of Barreiras-Bahia, through physical-chemical analysis. Five brands of inspected honeys were collected, obtained from supermarkets and markets in the city of Barreiras. There was a statistical difference $(\mathrm{P}<0.05)$ between the treatments for quantitative analysis. All samples showed water activity values below 0.60 , although this is not required by law, it is an important parameter to verify the free water content in the available product. All the samples of honeys were below $20 \%$ humidity, according to the legislation. The $\mathrm{pH}$ values in the analyzed samples varied from 3.79 to 4.16 and for acidity they were between 21.64 and $36.35 \mathrm{meq} / \mathrm{kg}$, thus complying with the legislation. The $\mathrm{pH}$ analysis can help in the evaluation of the honeys quality, together with the acidity analysis. The levels of reducing sugars obtained were between $70.95 \%$ and $73.50 \%$, already for apparent sucrose that according to Brazilian legislation recommends a maximum limit of $6 \%$ the values obtained varied between $1.85 \%$ and $4.40 \%$. Only the
\end{abstract}


MM5 show was inside the limits of (HMF), in relation to the diastatic activity, all were below the minimum recommended values. In relation to the qualitative analyses of Lund and Lugol, all the brands analyzed were not indicative of adulteration or bad conservation of honey. The 5 brands analyzed of honeys with (SIF) met the legislation on the following parameters: humidity, ashes, $\mathrm{pH}$, acidity, reducing sugar, apparent sucrose.

Keywords: Honey; Hydroxymethylfurfural; Diastatic activity; Industrial.

\section{Resumen}

Este estudio se desarrolló con el objetivo de evaluar la calidad de las mieles industrializadas (inspeccionadas) vendidas en la ciudad de Barreiras-Bahia, a través de análisis físico-químicos. Se recogieron cinco marcas de mieles inspeccionadas, obtenidas en supermercados y mercados de la ciudad de Barreiras. Hubo una diferencia estadística $(\mathrm{P}<0,05)$ entre los tratamientos para los análisis cuantitativos. Todas las muestras presentaron valores de actividad del agua inferiores a 0,60, aunque la legislación no lo exige, es un parámetro importante para verificar el contenido de agua libre en el producto disponible. Todas las muestras de miel estaban por debajo del $20 \%$ de humedad, según la legislación. Los valores de $\mathrm{pH}$ de las muestras analizadas variaron de 3,79 a 4,16 y para la acidez estuvieron entre 21,64 y $36,35 \mathrm{meq} / \mathrm{kg}$, cumpliendo así con la legislación. El análisis del pH puede ayudar en la evaluación de la calidad de las mieles, junto con el análisis de la acidez. Los niveles de azúcares reductores obtenidos estaban entre el $70,95 \%$ y el 73,50\%, mientras que para la sacarosa aparente, que según la legislación brasileña prescribe un límite máximo del 6\%, los valores obtenidos oscilaban entre el 1,85\% y el 4,40\%. Sólo la muestra MM5 estaba dentro de los límites del (HMF), en relación con la actividad diastásica, todos estaban por debajo de los valores mínimos recomendados. En cuanto a los análisis cualitativos de Lund y Lugol, todas las marcas analizadas no eran indicativas de adulteración o mala conservación de la miel. Las 5 marcas analizadas de mieles con (SIF) cumplieron los siguientes parámetros: humedad, cenizas, $\mathrm{pH}$, acidez, azúcar reductor, sacarosa aparente.

Palabras clave: miel; Hidroximetilfurfural; Actividad diastática; Industrial.

\section{Introdução}

O mel é um produto naturalmente produzido por abelhas e, devido a sua composição, possui sabor característico e alto valor nutritivo. Seu uso é relatado desde a antiguidade e, a cada dia ganha novos mercados consumidores (Ribeiro \& Starikoff, 2019).

O mel é um produto naturalmente produzido por abelhas melíferas e é obtido a partir do néctar das flores, de secreções de partes vivas de plantas ou de excreções de insetos sugadores de partes vivas de plantas (Brasil, 2000). Ainda de acordo com a mesma legislação brasileira, o mel é composto por uma solução de açúcares concentrados, principalmente glicose e frutose, os quais influenciam diretamente sua doçura, além de possuir enzimas (invertase, amilase e glico-oxidase), aminoácidos, ácidos orgânicos, minerais, pigmentos e grãos de pólen.

Muitos fatores, tais como as floras melíferas, natureza do solo, raça de abelhas, estado fisiológico da colônia, estágio de maturação do mel e condições meteorológicas, são responsáveis por alterações nas propriedades do mel, com variações em seu aroma, paladar, coloração, viscosidade e propriedades medicinais (Souza, 2017; Barbosa et al., 2016; Campos et al., 2000). Contudo, estas características também podem ser modificadas por adulterações geradas por fontes não confiáveis que fazem mau uso do produto, adicionando em sua composição substâncias de menor valor comercial e nutritivo. Isto ocorre, principalmente, devido ao fato do mel, como mercadoria, ter disponibilidade limitada e um preço relativamente alto, incentivando a sua adulteração (Ribeiro et al., 2009).

No entanto, essas fraudes e outras alterações que o mel pode apresentar, são facilmente detectadas por análises em laboratório. A obtenção de parâmetros físico-químicos de méis é importante para sua caracterização como também é primordial para garantir a qualidade desse produto no mercado (De Assis Carvalho et al., 2020; Barbosa et al., 2016).

A necessidade dos serviços fiscalização de produtos e no controle da qualidade do mel, e que abrangem os serviços de Defesa e Inspeção dos produtos de origem animal em nível federal (SIF), estadual (SIE) e municipal (SIM), para a certificação de qualidade e procedência do mel fundamenta-se em aspectos econômicos, devido às barreiras impostas por alguns mercados quanto à qualidade e originalidade dos produtos, de saúde pública, por causa dos efeitos deletérios que o mel fora dos padrões 
de qualidade pode causar aos consumidores, e legais, dada a possibilidade de embargo de unidades produtoras e confisco dos produtos no comércio (De Mello Sousa, et al., 2013).

A exigência por produtos de qualidade disponíveis ao consumo humano reflete a necessidade de uma maior fiscalização durante o processo de produção (Ribeiro \& Starikoff, 2019). No Brasil, as análises físico-químicas indicadas pela legislação para o controle de qualidade do mel puro são: umidade, acidez, cinzas, açúcares redutores e não-redutores, açúcares totais, pH, cor, sólidos insolúveis em água, atividade diastásica e hidróximetilfurfural (HMF) (Brasil, 2000).

A definição de parâmetros físico-químicos de amostras de méis é importante para sua caracterização, e primordial para garantir a qualidade deste produto no mercado, além de poder determinar o grau de maturidade, pureza e deterioração (Ribeiro et al., 2009; Carvalho et al., 2003).

Análises físico-químicas de méis brasileiros inspecionados têm sido a temática de pesquisas para monitorar a sua qualidade. Nesse sentido, muitos trabalhos têm sido desenvolvidos (De Assis Carvalho et al., 2020; Ribeiro \& Starikoff, 2019; Marinho et al., 2018; Dantas et al., 2017; Barbosa et al., 2016; Melo et al., 2016; De Mello Sousa, et al., 2013).

O presente estudo foi desenvolvido com o objetivo de avaliar a qualidade de méis industrializados (inspecionados) comercializados na cidade Barreiras-Bahia, através de análises físico-químicas.

\section{Metodologia}

A investigação visa trazer novos conhecimentos à sociedade, como declarado por Pereira et al. (2018). A pesquisa foi desenvolvida nos Laboratórios de Análise de Alimentos do Instituto Federal da Bahia - IFBA, campus Barreiras e de Bromatologia da Universidade Federal do Oeste da Bahia - UFOB, campus Barreiras, num estudo quantitativo e qualitativo.

Foram coletadas 5 marcas de méis inspecionados, sendo dois lotes de cada, totalizando 10 amostras analisadas. As amostras de méis foram obtidas em supermercados e mercados da cidade de Barreiras - Bahia, sendo posteriormente transportadas e conservadas sob temperatura ambiente, desde a compra até o momento das análises, sendo mantidas nas embalagens de origem e ao abrigo da luz solar até serem utilizadas para análises, a fim de evitar qualquer tipo de alteração.

Todas as marcas de méis adquiridas no comércio possuíam Selo de Inspeção, sendo 3 com Serviço de Inspeção Federal (SIF) e duas com Serviço de Inspeção Estadual (SIE). As amostras foram consideradas como tratamentos, sendo identificadas da seguinte maneira: MM1, MM2, MM3, MM4 e MM5.

Para verificar a qualidade do mel foram realizadas análises quantitativas: $\mathrm{pH}$, acidez titulável, atividade diastásica, açúcares redutores, sacarose aparente, hidroximetilfurfural (HMF), cinzas (minerais), e umidade. As análises de proteína e lugol foram realizadas de forma qualitativas. Todas as análises foram conduzidas, segundo metodologia de Adolfo Lutz (IAL, 2008).

O teste Lugol foi aplicado para avaliar a presença de amido nas amostras de mel, segundo o método 0184/IV, no qual $10 \mathrm{~g}$ de amostra foram diluídos em $20 \mathrm{~mL}$ de água destilada e levados à ebulição, seguidos de arrefecimento e depois adicionados de $0,5 \mathrm{~mL}$ de solução de Lugol. A presença de amido foi evidenciada por uma cor azul nas amostras.

A presença/ausência de proteína foi determinada por um teste qualitativo, a partir da adição de $1 \mathrm{~g}$ de mel, $15 \mathrm{~mL}$ de água e $5 \mathrm{~mL}$ de ácido tânico, que atua na formação do precipitado proteico, em proveta de $25 \mathrm{~mL}$. A solução foi deixada em repouso por 24 horas em temperatura ambiente, observando em seguida à matéria precipitada.

A atividade de água foi determinada em aparelho Aqualab CX2. Para a determinação das viscosidades dos méis foi utilizado um viscosímetro da marca Brookfield, modelo RVT.

As análises estatísticas foram realizadas com duas repetições. Os dados foram interpretados por meio da análise de variância (ANOVA) e, quando necessário, as médias foram comparadas utilizando-se o teste de Tukey, a 5\% de probabilidade. 
As análises estatísticas foram realizadas no software Statistical Analysis System (SAS) (SAS Institute Inc., Cary, NC, USA), licenciado para a Universidade Federal de Lavras (UFLA).

\section{Resultados e Discussão}

As variáveis apresentadas na Tabela 1: atividade de água (Aw), umidade, cinzas e viscosidade, apresentaram diferenças significativas $(\mathrm{P}<0,05)$ entre os tratamentos, nas amostras de méis inspecionados comercializadas na cidade de Barreiras- BA.

A Normativa $n^{\circ}$ 011/2000 (Brasil, 2000), não determina como obrigatoriedade a análise para Atividade de água (Aw) em mel, porém é um importante parâmetro para verificar o teor de água livre no produto disponível, passando a ser um forte aliado como ferramenta para se verificar fraude ou adulterações do produto. De acordo com a Tabela 1, todas as amostras apresentaram valores de atividade de água abaixo de 0,60 .

Os valores obtidos para determinação de atividade de água em amostras de mel de marcas comerciais adquiridas em estabelecimentos no município de Ponta Grossa-PR, variaram entre 0,62 a 0,58, conforme Silva, (2016).

Tabela 1. Valores médios de parâmetros físico-químicos das análises de atividade de água, umidade, cinzas e viscosidade de amostras de méis inspecionados, comercializadas em mercados e supermercados.

\begin{tabular}{ccccc}
\hline AMOSTRAS & \multicolumn{4}{c}{ PARÂMETROS FÍSICO-QUÍMICOS } \\
\cline { 2 - 5 } DE MÉIS & Aw & Umidade (\%) & Cinzas (\%) & Viscosidade (cP) \\
\hline MM1 & $0,565^{b}$ & $16,9^{b}$ & $0,224^{b}$ & $9.066^{b}$ \\
MM2 & $0.580^{a}$ & $17,7^{a}$ & $0,166^{d}$ & $3.872^{e}$ \\
MM3 & $0,562^{c}$ & $17,1^{b}$ & $0,225^{b}$ & $5.224^{c}$ \\
MM4 & $0,551^{d}$ & $16,5^{c}$ & $0,205^{c}$ & $4.616^{d}$ \\
MM5 & $0,565^{b}$ & $17,1^{b}$ & $0,497^{a}$ & $14.630^{a}$ \\
\hline
\end{tabular}

Médias seguidas pela mesma letra não diferem $(\mathrm{P}>0,05)$ entre si pelo teste de Tukey na mesma coluna. Fonte: Autores (2019).

A umidade constitui um dos principais parâmetros relacionados à qualidade do mel influenciando diretamente em outras características como o sabor, a viscosidade, a conservação a palatabilidade, entre outros (Vieira et al., 2017; Persanooddo; Piro, 2004). Os valores de umidade estabelecidos pela Legislação Brasileira para mel é de até $20 \%$ ou 20 g de umidade/ 100g de mel analisado (BrasiL, 2000). Todas as amostras atenderam a legislação para esse parâmetro (Tabela 1).

Gomes et al. (2015) obtiveram resultados de umidade, para sete amostras do município de Soure, Arquipélago de Marajó, uma variação de 13,26\% a 18,26\%, enquadrando todas as amostras dentro da legislação.

Para o teor de cinzas os valores encontrados variaram de 0,551 a 0,580 (Tabela 1). Os valores obtidos para cinzas neste estudo estão em conformidade com a legislação vigente, que determina para esse parâmetro índices aceitáveis de até 0,6\% (Brasil, 2000). O conteúdo de cinzas ou minerais no mel está diretamente relacionado às condições ambientais e às origens botânicas e geográficas (Karabagias et al., 2014).

As amostras analisadas tiveram variação do valor médio do coeficiente de viscosidade entre $3.872 \mathrm{cP}$ (centipoise) a $14.630 \mathrm{cP}$. A viscosidade do mel remete a sua densidade relativa, logo, quanto menor o conteúdo de água, maiores os índices de densidade e viscosidade. Apesar de ser um parâmetro importante em relação à qualidade do mel, não possui critério de avaliação para viscosidade nas legislações vigente (Alves et al., 2006). 
A amostra MM5 (Tabela 1) apresentou um alto índice da média do coeficiente de viscosidade (14.630 cP), e entre as amostras analisadas foi a única que apresentou início do processo de cristalização, que pode ocorrer naturalmente no mel, pelo alto teor de glicose, este que indica colheita do mel antes do completo período de maturação.

Em relação as análises físico-químicas de acidez titulável, $\mathrm{pH}$, açúcar redutor e sacarose aparente de méis inspecionados comercializadas na cidade de Barreiras- BA, os resultados obtidos nas estão apresentados nas Tabela 2. Para todos os parâmetros citados, houve efeito significativo entre as amostras $(\mathrm{P}<0,05)$, de acordo com a Tabela 2.

Tabela 2. Valores médios de parâmetros físico-químicos das análises de $\mathrm{pH}$, acidez titulável, açúcar redutor e sacarose aparente de amostras de méis inspecionados, comercializadas na cidade de Barreiras - Bahia.

\begin{tabular}{ccccc}
\hline AMOSTRAS & \multicolumn{3}{c}{ PARÂMETROS FÍSICO-QUÍMICOS } \\
\cline { 2 - 5 } DE MÉIS & $\mathbf{p H}$ & $\mathbf{A T}(\mathbf{m E q} / \mathbf{K g})$ & Açúcar redutor (\%) & Sacarose aparente (\%) \\
\hline MM1 & $3,95^{c}$ & $21,64^{e}$ & $73,50^{a}$ & $2,65^{c}$ \\
MM2 & $3,79^{d}$ & $36,35^{a}$ & $72,30^{b}$ & $2,50^{d}$ \\
MM3 & $4,03^{b}$ & $28,03^{b}$ & $71,55^{c}$ & $4,40^{a}$ \\
MM4 & $4,16^{a}$ & $25,16^{c}$ & $70,95^{d}$ & $3,75^{b}$ \\
MM5 & $4,15^{a}$ & $25,07^{d}$ & $70,95^{d}$ & $1,85^{e}$ \\
\hline
\end{tabular}

Médias seguidas pela mesma letra não diferem $(\mathrm{P}>0,05)$ entre si pelo teste de Tukey na mesma coluna.

Fonte: Autores (2019).

O valor de $\mathrm{pH}$ considerado ideal para o mel varia de 3,3 a 4,6 (BrasiL, 2000). Os valores de pH nas amostras analisadas, variavam de 3,79 a 4,16 (Tabela 2), estando, portanto, dentro da faixa estabelecida pela legislação. A análise de pH pode auxiliar na avaliação da qualidade de méis, em conjunto com a análise de acidez (Marinho et al., 2018).

Ribeiro e Starikoff, (2019), avaliaram a qualidade físico-química de 22 amostras de méis com registro de inspeção e de comércio informal de diferentes municípios da região Sul do Brasil, e constataram que 90\% das amostras estão desacordo com o limite padrão que vai de 3,30 a 4,60.

Silva, (2016), ao analisar sete amostras de mel, de marcas comerciais adquiridas em estabelecimentos comerciais no município de Ponta Grossa-Paraná, obteve valores de pH variando entre 4,14 a 4,62. Lirio et al., (2015) ao analisarem 24 amostras de méis silvestres comercializados no Estado do Rio de Janeiro, obtiveram resultados para pH entre 3,80 a 4,90.

Pela Legislação vigente, o mel não deve ultrapassar o valor de 50 miliequivalentes de acidez/ kg de mel (Brasil, 2000). Os resultados dos méis variaram entre 21,64 e 36,35 meq/kg, atendendo assim a legislação.

A acidez do mel é um compo-nente de extrema relevância, pois além de conferir características químicas e sensoriais, contribui para a sua estabilidade frente ao desenvolvimento de microrganismos, assim como permitem indicar más condições de manuseio e armazenamento que resultaram em processos fermentativos (Marinho et al., 2018).

Ribeiro et al., (2009), observaram que das 25 amostras de méis inspecionadas no estado do Rio de Janeiro, 12\% apresentaram inconformidade em relação a acidez, com valores de 14 a $69 \mathrm{meq} / \mathrm{kg}$. Analisando méis inspecionados comercializados em supermercados na cidade de Sete Lagoas - Minas Gerais, Barbosa et al, (2016) obtiveram médias de 27,73 $\mathrm{meq} / \mathrm{kg}$ de acidez.

Os teores de açúcares redutores obtidos ficaram entre 70,95\% a 73,50\% (Tabela 2), e a Instrução Normativa $n^{\circ}$ 11(Brasil, 2000) estabelece que o mel floral deve apresentar no mínimo $65 \mathrm{~g} \cdot(100 \mathrm{~g})$-1 de glicídios redutores portanto, todas as amostras analisadas encontram-se dentro dos valores exigido pela legislação. 
Melo e Queiroz, (2016), verificaram a qualidade de amostras de mel inspecionados na região de Uberlândia e notaram que as 4 amostras analisadas atenderam a legislação para a quantidade de açúcares redutores.

A sacarose representa 2 a 3\% dos carboidratos do mel. Valores superiores a este limite podem indicar que o mel foi extraído prematuramente ou sofreu algum tipo de adulteração (Ribeiro et al., 2009). A legislação brasileira preconiza um limite máximo de 6\% (Brasil, 2000). Este limite foi respeitado em todas as cinco amostras de méis inspecionados comercializados na cidade de Barreiras, que apresentaram valores variando de $1,85 \%$ a $4,40 \%$,

Ribeiro et al., (2009), analisaram 25 amostras de méis inspecionadas no estado do Rio de Janeiro, obtendo valor médio de 69,2\% para açúcares redutores e 2,5\% para sacarose. Barbosa et al, (2016) obtiveram médias de 80,24\% de açúcar redutor e 3,78\% para sacarose aparente, em amostras de mel comercializados em supermercados na cidade de Sete Lagoas Minas Gerais.

Os níveis de hidroximetilfurfural e a atividade diastásica também foram determinados nas amostras de méis inspecionados, considerando a importância destes parâmetros na avaliação da qualidade do mel. Houve diferenças significativa $(\mathrm{P}<0,05)$ entre os tratamentos, para os dois parâmetros. Os resultados obtidos são apresentados na Tabela 3.

A Legislação Brasileira regulamenta que para manter o Padrão de Qualidade e Identidade do mel comercializado, o limite estabelecido de Hidroximetilfurfural (HMF) deve ser equivalente à até $60 \mathrm{mg} / \mathrm{Kg}$ (Brasil, 2000). De acordo com os dados apresentados na Tabela 3, apenas a amostra MM5 estava em conformidade com a legislação.

Quando o mel é armazenado em condições adequadas, ou seja, ao abrigo de luz e temperaturas de até $20^{\circ} \mathrm{C}$, os níveis de HMF permanecem aceitáveis. Provavelmente as demais amostras (Tabela 3), que apresentaram quantidade de HMF acima de $60 \mathrm{mg} / \mathrm{kg}$, estavam fora do padrão por terem sido submetidas a longo tempo de estocagem e/ou superaquecimento, que são fatores que contribuem para a formação do HMF (Granato \& Nunes, 2017).

Tabela 3. Valores médios de parâmetros físico-químicos das análises de atividade diastásica e hidroximetilfurfural de amostras de méis inspecionados, comercializadas na cidade de Barreiras - Bahia.

\begin{tabular}{ccc}
\hline AMOSTRAS & \multicolumn{2}{c}{ PARÂMETROS FÍSICO-QUÍMICOS } \\
\cline { 2 - 3 } DE MÉIS & Atividade diastásica & Hidroximetilfurfural (mg/kg) \\
\hline MM1 & $4,3^{b} \pm 0,2$ & $253,3^{a}$ \\
MM2 & $6,8^{a} \pm 0,1$ & $132,4^{b}$ \\
MM3 & $3,9^{d} \pm 0,1$ & $108,6^{c}$ \\
MM4 & $2,1^{e} \pm 0,1$ & $64,2^{d}$ \\
MM5 & $4,2^{c} \pm 0,1$ & $42,2^{e}$ \\
\hline
\end{tabular}

Médias seguidas pela mesma letra não diferem $(\mathrm{P}>0,05)$ entre si pelo teste de Tukey na mesma coluna. Fonte: Autores (2019).

Analisando a qualidade de 62 marcas de méis inspecionados, no Rio de Janeiro, De Mello Sousa et al. (2013), encontraram 35,65 mg/Kg como valor médio de HMF, atendendo, portanto, aos requisitos da legislação.

A legislação brasileira estabelece que a atividade diastásica deve apresentar um mínimo de oito unidades na escala Gothe (Brasil, 2000). Conforme exposto na Tabela 3, os valores encontrados variaram entre 2,1 e 6,8 unidades Gothe. Assim, todas as amostras atingiram resultados inferiores a esse valor. Valores de diástases diminuídos estão correlacionados ao processo de desnaturação das amilases, ocorrido durante um possível tratamento térmico durante seu processamento (CRANE 1987). 
Carneiro et al. (2015) ao pesquisarem amostras de méis inspecionados e informais constataram que 61,82\% das amostras estavam em desacordo com a legislação. O valor mínimo para atividade diastásica foi de 0 unidades Gothe e o valor máximo foi de 22,7 unidades Gothe.

Silva et al. (2011) obtiveram médias de 15,30 unidades Gothe, para méis com registro de inspeção. Lirio et al. (2015) obtiveram média de 8,8 unidades Gothe e, valores mínimos e máximos para atividade diastásica de 0,8 e 37,8 unidades Gothe.

As reações de Lund e Lugol são consideradas análises qualitativas e complementares que podem indicar adulteração ou má conservação do mel.

Tabela 4. Resultados qualitativos de parâmetros químicos das análises de Reação Lund e Lugol de amostras de amostras de méis inspecionados, comercializadas na cidade de Barreiras - Bahia.

\begin{tabular}{cccccc}
\hline PARÂMETROS & \multicolumn{5}{c}{ AMOSTRAS DE MÉIS } \\
\cline { 2 - 6 } ANALISADOS & MM1 & MM2 & MM3 & MM4 & MM5 \\
\hline Reação de Lund & + & + & + & + & + \\
Reação de Lugol & - & - & - & - & - \\
\hline
\end{tabular}

Os símbolos (-) e (+), indicam ausência e presença ou negativo e positivo, respectivamente, conforme cada análise apresentada na tabela. Fonte: Autores (2019).

Ribeiro e Starikoff, (2019), observaram que na reação de Lund, 9,09\% das amostras não formaram precipitado, caracterizando amostras de méis adulterados. Cardoso Filho et al. (2011), ao analisarem 16 amostras de méis comercializados em diferentes municípios do Mato Grosso do Sul, constataram que 50\% das amostras analisadas para a reação de Lund não apresentaram a formação de precipitado.

Em estudo realizado em Salvador-BA, Leal, Silva e Jesus (2001) perceberam que 30\% das amostras de méis comercializadas, não formaram precipitados, estando em desacordo com a legislação brasileira.

A reação de Lugol está relacionada à pureza do mel, utilizando iodo e iodeto de potássio (Lugol), que na presença de amido e dextrinas no mel é responsável pela formação de cor característica (IAL, 2008). De acordo com a Tabela 4, todas as amostras não apresentaram coloração avermelhada, sendo consideradas negativas para Reação de Lugol, indicando assim que amostras de méis inspecionadas comercializadas na cidade Barreiras não foram adulteradas com adição de amido.

Do Santos et al. (2015), ao analisarem cinco amostras de méis do município de Itaquera, SP, observaram que para a reação de Lugol, duas amostras foram positivas. Em 25 amostras de méis inspecionadas no estado do Rio de Janeiro, Ribeiro et al. (2009), verificaram que 3 amostras apresentaram coloração marrom-avermelhada para a reação de Lugol, sendo consideradas positivas, o que indica adição de amido.

Os valores encontrados nesse trabalho estão compatíveis aos sugeridos por Da Silva Dias et al., 2009, ao analisarem a qualidade de três amostras de méis industrializados comercializados em Londrina -Paraná.

\section{Considerações Finais}

As 5 marcas analisadas de méis com selo de Serviço de Inspeção Federal (SIF) atenderam ao Regulamento técnico de identidade e qualidade do mel nos seguintes parâmetros: umidade, cinzas, $\mathrm{pH}$, acidez, açúcar redutor, sacarose aparente.

Os resultados obtidos mostram que $100 \%$ das amostras inspecionadas estão em desacordo com as especificações da legislação para a qualidade do mel em relação a atividade diastásica, enquanto que $80 \%$ das amostras de méis apresentaram-se fora dos padrões estabelecidos pela análise de hidroximetilfurfural, embora não signifique adulteração do produto e sim peculiaridades dos méis de marcas diferentes, indicativo de aquecimento ou longa estocagem. 
Em relação as análises qualitativas de Lund e Lugol, todas as marcas analisadas não foram indicativas de adulteração ou má conservação do mel.

Como sugestões para trabalhos futuros, deve ser feito avaliação das propriedades físico-químicas do mel comercializado em outras cidades a fim de verificar possíveis adulterações. Recomenda-se o uso de métodos instrumentais, especialmente os baseados em cromatografia e técnicas de espectroscopia, para identificar os tipos de adulterações.

\section{Agradecimentos}

Os autores agradecem ao Instituto Federal de Educação, Ciência e Tecnologia da Bahia, Campus Barreiras, por seu apoio financeiro.

\section{Referências}

Alves, J. E., \& Freitas, B. M. (2006). Comportamento de pastejo e eficiência de polinização de cinco espécies de abelhas em flores de goiabeira (Psidium guajava L.). Revista Ciência Agronômica, 37(2), 216-220.

Barbosa, S., Ribeiro, J. L. \& Ubaldo, J. C. S. R. Características físico-químicas de méis comercializados na cidade de Sete Lagoas-MG. In: XXV Congresso Brasileiro De Ciência E Tecnologia De Alimentos, n 25, 2016.

Bera, A., \& Almeida-Muradian, L. B. D. (2007). Propriedades físico-químicas de amostras comerciais de mel com própolis do estado de São Paulo. Food Science and Technology, 27(1), 49-52.

Campos, G., Nappi, U., Raslan, D., \& Augusti, R. (2000). Substâncias voláteis em mel floral e mel de melato. Ciência e Tecnologia de Alimentos, 20(1), 1822.

Cardoso Filho, N., Coelho, R. M., Rodrigues, A., Miguel, R. M., \& Camargo, T. R. C. (2011). Avaliação físico-química de méis comercializados em algumas cidades do Estado de Mato Grosso do Sul. Ensaios e Ciência: Ciências Biológicas, Agrárias e da Saúde, 15(6), 135-146.

Carneiro D. S. et al. Qualidade físico-química de méis comercializados na Cidade de Recife, Pernambuco. In: XXV Congresso Brasileiro de Zootecnia: ZOOTEC, 2015.

Carvalho, C. A. L., Alves, R. M. O., \& Souza, B. A. (2003). Criação de abelhas sem ferrão: aspectos práticos. Série meliponicultura, No. 1. UFBA/SEAGRIBA, Bahia, Brazil.

Crane E. (1987). O livro do mel. Editora Nobel. 226p.

Da Silva Dias, J., de Camargo, A. C., Barin, C. S., \& Ellensohn, R. M. (2009). Caracterização Físico-Química de Amostras de Mel. UNOPAR Científica Ciências Exatas e Tecnológicas, 8(1).

Dantas, M. C. D. A. M., do Nascimento Silva, S., Gomes, D. J., Neto, J. F., de Lima, C. J., \& da Silva, R. A. (2017). Avaliação da qualidade físico-química e microbiológica de méis de abelhas obtidos no comércio de Sousa, Paraíba. ACTA Apicola Brasilica, 5(1), 01-05.

De Assis Carvalho, R., Ribeiro, A. C., Lima, C. M., da Silva Mariz, W. P., Silva, L. S., da Silva, A. M., \& Trombete, F. M. (2020). Assessment of adulteration and mycoflora identification of honey samples marketed in the metropolitan region of Belo Horizonte, Brazil. Research, Society and Development, 9(7), 440974246 .

De Mello Sousa, J. P. L., Koshiyama, A. S., de Souza Tassinari, W., Sant'Ana, L. D. O., Castro, R. N., Lorenzon, M. C. A., \& Keller, K. M. (2013). Indicadores físico-químicos de qualidade de méis de apis mellifera 1. e suas relações com o selo de inspeção animal. Brazilian Journal of Veterinary Medicine, 35(3), 236-240.

Dos Santos, A. B., de Moura, C. L., \& Camara, L. B. (2015). Determinação da autenticidade dos méis vendidos nas feiras livres e comércios populares. Brazilian Educational Technology: research and learning, 2(3).

Gomes, P. W. P., Muribeca, A. D. J. B., Silva, M. D. B., Carneiro, J. S., \& Souza, R. F. (2015). Avaliação físico-química e microbiológica de méis de abelhas comercializados no município de Soure-Marajó-Pará. Enciclopédia Biosfera, Centro Científico Conhecer, Goiânia, 11(22), 2920.

Granato, D., \& Nunes, D. (2017). Análises químicas, propriedades funcionais e controle de qualidade de alimentos e bebidas: uma abordagem teóricoprática. Elsevier Brasil.

Instituto Adolfo Lutz. (2008). Métodos físico-químicos para análise de alimentos: Instituto Adolfo Lutz.

Karabagias, I. K., Badeka, A., Kontakos, S., Karabournioti, S., \& Kontominas, M. G. (2014). Characterisation and classification of Greek pine honeys according to their geographical origin based on volatiles, physicochemical parameters and chemometrics. Food chemistry, $146,548-557$.

Leal, V. M., Silva, M. H., \& Jesus, N. M. (2001). Aspecto físico-químico do mel de abelhas comercializado no município de Salvador- Bahia. Revista Brasileira de Saúde e Produção Animal, 1(1),14-18. 
Research, Society and Development, v. 10, n. 1, e28710110959, 2021 (CC BY 4.0) | ISSN 2525-3409 | DOI: http://dx.doi.org/10.33448/rsd-v10i1.10959

Lirio, F. C., dos Santos Bello, M., Moura, M. R. L., de Carvalho, L. M. J., \& Gregorio, S. R. (2015). Avaliação dos parâmetros físico-químicos e análise por componentes principais de méis silvestres produzidos e comercializados no Estado do Rio de Janeiro, Brasil. Infarma-Ciências Farmacêuticas, 27(3), 168175.

Marchini, L. C., Moreti, A. C. D. C. C., \& Otsuk, I. P. (2005). Análise de agrupamento, com base na composição físico-química, de amostras de méis produzidos por Apis mellifera L. no Estado de São Paulo. Food Science and Technology, 25(1), 8-17.

Marchini, L. C., Moreti, A. C. D. C. C., \& Otsuk, I. P. (2005). Análise de agrupamento, com base na composição físico-química, de amostras de méis produzidos por Apis mellifera L. no Estado de São Paulo. Food Science and Technology, 25(1), 8-17.

Marinho, J. K. L., Moreira, C. V. D. S., Ferreira, L. C., Damasceno, K. S. F. D. S. C., Santos, J. A. B. D., \& Holland, N. (2018). Avaliação da qualidade físicoquímica e microbiológica de méis comercializados em Natal, RN. Rev. Inst. Adolfo Lutz, 1-6.

Melo, C. M. T., Silva, V., \& Queiroz, C. (2016). Características físico-químicas do mel comercializado na região de Uberlândia. Ambiência Guarapava, 12(12), 739-763.

Ministério da Agricultura, Pecuária e Abastecimento, \& BRASIL. Ministério da Agricultura, Pecuária e Abastecimento. (2000). Instrução Normativa n ${ }^{11}$, de 20 de Outubro de 2000: Aprova o Regulamento Técnico de Identidade e Qualidade do mel. Diário Oficial [da] República Federativa do Brasil.

Pereira, A. S., Shitsuka, D. M., Parreira, F. J,. \& Shitsuka, R. (2018). Metodologia da pesquisa científica. Ed. UAB/NTE/UFSM. https://repositorio.ufsm.br/bitstream/handle/1/15824/Lic_Computacao_Metodologia-Pesquisa-Cientifica.pdf?sequence=1. Pitt, J. I., \& Hocking, A. D. (2009). The Ecology of Fungal Food Spoilage. Fungi and Food Spoilage, 3-9.

Persano-Oddo L, Piro R (2004) Principais méis uniflorais europeus: folhas descritivas. Apidologie 35: 38-81.

Ribeiro, R. D. O. R., da Silva, C., Monteiro, M. L., Baptista, R. F., Guimarães, C. F., Mársico, E. T., \& da Silva Pardi, H. (2009). Avaliação comparativa da qualidade físico-química de méis inspecionados e clandestinos, comercializados no estado do Rio de Janeiro, Brasil. Revista Brasileira de Ciência Veterinária, 16(1).

Ribeiro, R., \& Starikoff, K. R. (2019). Avaliação da qualidade físico-química e microbiológica de mel comercializado. Revista de Ciências Agroveterinárias, 18(1), 111-118.

Silva, A. P. P. D. (2016). Determinação de identidade e qualidade em méis comercializados na região de Ponta Grossa-PR (Bachelor's thesis, Universidade Tecnológica Federal do Paraná).

Silva, M. B. L., Chaves, J. B. P., Valente, M. E. R., Gomes, J. C., \& Oliveira, G. F. (2011). Qualidade de méis produzidos por apicultores e méis provenientes de entrepostos registrados no Serviço de Inspeção Federal. Arquivo Brasileiro de Medicina Veterinária e Zootecnia, 63(4), $1043-1045$.

Souza, L. B. D. S. (2017). Caracterização físico-química e microbiológica do mel de abelhas (Apis mellifera) produzido no território rural de identidade Parque das Emas-Goiás. Dissertação (Mestrado em Tecnologia de Alimentos), Cidade: Instituto Federal Goiano. 60 p.

Vieira, G. H. C.; Gomes, M. F. F.; Moraes, A. N., Oliveira, A. F. (2017). Caracterização físico-química de méis produzidos no estado de Mato Grosso do Sul, Brasil. Revista de Agricultura Neotropical,4 (3), 30-34. 